\title{
Searching for target letters in memory: Individual preferences and instructions for text representation
}

\author{
VIVIAN I. SCHNEIDER, ALICE F. HEALY, and DAVID J. STEINHART \\ University of Colorado, Boulder, Colorado
}

\begin{abstract}
Participants searched for target letters in a short passage held in memory. In Experiment 1, participants were divided into two groups on the basis of a retrospective report concerning the type of representation used to store the passage in memory, and in Experiment 2, participants were instructed concerning the form of memory representation to use. Only participants using a visual representation missed more targets in the word the than in other words. Participants instructed to form a visual representation also made fewer content-word or phrase substitutions when learning the passage than did participants instructed to form an auditory representation. These findings show that choice of memory representation is flexible and that the representation used influences what can be retrieved from memory.
\end{abstract}

In earlier work (see Schneider, Healy, Ericsson, \& Bourne, 1989), we used a version of the letter-detection task as a means to illuminate memory representations of text material. Specifically, participants memorized short texts and then searched their memory for target letters in the texts, writing in sequence every word that contained a target. This memory task was compared with visual and auditory baseline tasks in which participants either read or heard texts and wrote down every word containing a target. For the visual baseline task, participants showed the typical signs of unitization found with the standard procedures; that is, they missed more targets in the very common word the than in other, less common words (see, e.g., Healy, 1976, 1994). In contrast, there was no difference in errors made on the word the and other words for the auditory baseline task. We found striking individual differences in the pattern of errors on the memory task, and these differences were related to participants' retrospective verbal reports concerning the representation they used to store the text. Those participants who said that they searched a visual (i.e., written) representation of the text in memory ( $32 \%$ of the total) showed the standard unitization pattern (i.e., more errors on the word the than on other words), whereas those participants who said that they searched an auditory (i.e., spoken) representation of

This research was supported in part by Army Research Institute Contract MDA903-93-K-0010 to the University of Colorado (A.F.H., principal investigator). We are indebted to James Parker for testing some of the participants in Experiment 2, to Arthur Glenberg, Marilyn Smith, and Henry Roediger III, for helpful comments on an earlier version of this manuscript, and to Lyle Bourne and William Marmie for helpful suggestions regarding this research. Correspondence should be addressed to V. I. Schneider, Department of Psychology, University of Colorado, Muenzinger Bldg., Boulder, CO 80309-0345 (e-mail: schneider@clipr.colorado.edu). the text in memory ( $48 \%$ of the total) showed an error pattern similar to that found in the auditory baseline condition (i.e., no more errors on the word the than on other words).

Two questions arise from these findings: (1) Are the individual differences we observed related to variations in visual imagery ability? This question is of interest because visual imagery ability has been shown to influence performance in a wide range of cognitive tasks (see Allen, Wallace, \& LoSchiavo, 1994, for a recent example). (2) Is a given participant able to search either type of representation, and is the type of representation used by a participant sensitive to instructions or other aspects of the experimental context? This question is of interest because one representation may be more efficient than another for some tasks so that instructions regarding the use of a particular representation could enhance task performance.

In Experiment 1, we gathered converging evidence for individual differences in memory representation for searching text, and we employed a standard test of visual imagery (Marks, 1973) to determine if individuals who score high on visual imagery are also those who would both give verbal reports of using a visual memory representation and show the pattern of detection errors consistent with the visual baseline condition.

In Experiment 2, we varied the instructions given to participants concerning the type of memory representation they should employ and determined whether individuals instructed to use a visual representation, but not those instructed to use an auditory representation, would show the pattern of detection errors consistent with the visual baseline condition.

\section{EXPERIMENT 1}

Participants were given a short passage to memorize across five successive study-test presentations. They were 
then asked to search their memory representation and write down every word containing the target letter $h$.

We divided participants into two groups depending on their retrospective report concerning memory type-auditory and visual. As in our earlier study (Schneider et al., 1989), we expected participants in the visual group, but not those in the auditory group, to show the typical signs of visual unitization-namely, more detection errors on the common word the than on other words containing an $h$.

We also made an alternative division of the participants into two groups on the basis of a standard test of visual imagery ability (Marks, 1973) - high and low visual imagery ability. If visual imagery ability affects memory representation, participants with high visual imagery ability should be more likely than those with low visual imagery ability to use a visual memory representation. Also, we would expect participants with high visual imagery ability, but not those with low visual imagery ability, to show the typical signs of visual unitization.

In our earlier study (Schneider et al., 1989), we employed both visual and auditory presentation modalities, but we found no differences in the memory representation participants used as a function of the presentation modality. Therefore, in the present study, the passage to be memorized was always presented visually to the participants.

\section{Method}

Participants. The participants were 16 students from introductory psychology classes at the University of Colorado. ${ }^{1}$ All of them were native English speakers who showed that they had fully memorized the passage by the fifth repetition.

Materials and Procedure. Participants were tested in small groups. They were given the same passage and packet of instructions as those used by Schneider et al. (1989). The passage read: “The boys' mother cleaned their clothes the other day. There was soap on the floor. Then Bob's brother slipped on the slick lather and fell." This passage is 25 words long and includes 12 test words with one instance of the letter $h$ in each; 4 of the test words are instances of the word the. The participants' packet included a page with the passage and instructions to read it. On the next page were instructions to write down as much of the passage as could be remembered. These two pages were repeated five times, thus constituting five memorization trials. Then there were instructions to recall the passage and write down all the words containing the letter $h$. The next page asked the participants to describe what they were remembering as the search task was being completed. The following page gave three examples and asked the participants to check what they had done:

1. Visualize the words in the passage, as if reading.

2. Visualize the scene of the passage, seeing the action.

3 . Say or hear the words in your mind.

Following the letter-detection packet was a two-page Vivid Visual Inventory Questionnaire (VVIQ) from Marks (1973); it included 16 items (e.g., the exact contour of face, head, shoulders, and body of a relative or friend), each of which was to be rated as to the vividness of the image that could be generated. The rating scale ranged from 1 ( perfectly clear and as vivid as normal vision) to 5 (no image at all, you only "know" that you are thinking of the object).

\section{Results}

The participants were divided into two groups on the basis of their responses on the second page of the proto- col questionnaire. There were 8 participants in the auditory and 8 in the visual imaging group.

A two-way mixed analysis of variance (ANOVA) was conducted on the proportion of detection errors made out of the total possible, including the between-subjects factor of memory report (auditory, visual) and the withinsubject factor of word type (the, other). The main effect of word type was significant $\left[F(1,14)=11.33, M S_{\mathrm{e}}=\right.$ $.0156, p<.005]$, with a greater proportion of errors on the $(M=.22)$ than on other words containing $h(M=.07)$. There was no main effect of memory report $(F<1)$. However, importantly, the interaction of the two factors was significant $\left[F(1,14)=5.30, M S_{\mathrm{e}}=.0156, p<.05\right]$. To explore this interaction, two planned one-way ANOVAs were conducted on the auditory and visual processing groups. As expected, for participants who reported using an auditory method of memory search, there was no difference between the proportion of errors made on the and on other words $[F(1,7)<1]$, whereas for participants who reported using a visual search method, a significantly greater proportion of errors was made on the than on other words $\left[F(1,7)=12.44, M S_{\mathrm{e}}=.0201, p<.01\right]$; see Table 1.

The range of scores for the VVIQ was 1.31 to 3.00 , with 5 , which is the highest possible score, indicating low visual imagery. The participants were divided by a median split into high- and low-VVIQ groups based on their mean scores, with 8 participants in each group. Four participants used a visual search method and 4 used an auditory search method in the high-VVIQ group, and 4 participants used a visual search method and 4 used an auditory search method in the low-VVIQ group. A threeway mixed factorial ANOVA was conducted on the pro. portion of errors made out of the total possible, including the between-subjects factors of memory report and VVIQ group and the within-subject factor of word type. There were no significant effects involving VVIQ group.

\section{Discussion}

The present results confirmed our earlier findings (Schneider et al., 1989) that participants who reported using visual memory to search the passage, but not those who reported using auditory memory, showed the typical visual unitization effect for letter-detection errorsnamely, more errors on the common word the than on other test words.

Table 1

Proportion of Errors in Experiments 1 and 2 as a Function of Word Type and Memory Report (Experiment 1) or Instruction Group (Experiment 2)

\begin{tabular}{ccc}
\hline & \multicolumn{2}{c}{ Word Type } \\
\cline { 2 - 3 } Condition & The & Other Words \\
\hline & Experiment 1 \\
Memory report & .28 & .03 \\
Visual & .16 & .11 \\
Auditory & Experiment 2 & \\
& & \\
Instruction group & .12 & .06 \\
Visual & .02 & .03 \\
Auditory & & \\
\hline
\end{tabular}


We did not confirm, however, the hypothesis that use of visual memory was related to visual imagery ability, because participants with high visual imagery ability were no more likely than those with low visual imagery ability to use a visual search method or to show the typical visual unitization effect. Participants might choose to use an auditory memory representation for the task even if their visual imagery ability is high, because individuals who are high in visual imagery ability may also be high in auditory imagery ability.

\section{EXPERIMENT 2}

The memory representation used by participants is not a function of their visual imagery ability. The question remains whether the representation used is fixed for a given individual, determined by some ability or trait other than visual imagery, or whether, instead, the representation used by a given individual is flexible, determined by the context or other aspects of the particular memory task. To address this question, we varied the instructions given to participants in Experiment 2. Participants were divided into auditory and visual instruction groups. If the memory representation is flexible, participants in the visual instruction group should show the pattern of letterdetection errors found for participants who report using a visual memory representation, and participants in the auditory instruction group should show the pattern found for participants who report using an auditory memory representation.

\section{Method}

Participants. The participants were 24 students from the introductory psychology classes at the University of Colorado. ${ }^{2}$ The participants were assigned to instruction conditions and counterbalancing subconditions according to a fixed rotation on the basis of their seat location in the testing room. All of them met the following criterion for memorization of each passage (which was relaxed from the full memorization criterion used in Experiment 1 because we required participants to memorize two passages): Any errors on the fifth memorization trial did not involve a test word.

Materials and Procedure. The packet of instructions for this experiment was similar to the one used for Experiment 1, with a few changes. Participants memorized two passages in counterbalanced order and searched memory for 2 targets in counterbalanced order. Two passages were used. Passage A was the same as that in Experiment 1. Passage B, like Passage A, was used previously by Schneider et al. (1989); it read: "The busy mother gave the messy babies a quick bath Then the father laid them carefully in the feather bed with their fluffy brown bear." Both passages are 25 words long and include 12 instances of each target letter $(t$ and $h)$. In both passages the 2 targets always occur together in the sequence $t h ; 4$ of them are instances of the word the

The participants given the auditory instructions were told initially "to remember the passage by forming an auditory image of the words in the passage as if you are listening to the passage from a tape-recording in your mind. That is, try to "hear' the words in your mind." At the time of recall, they were also told to "try to recall the passage to yourself by forming an auditory image of the words in the passage as if you are listening to the passage from a tape-recording in your mind." The participants given the visual instructions were told instead to remember and recall the passage "by forming a visual image of the words in the passage as if you are reading the passage from a piece of paper in your mind." They were told to "try to 'see' the words in your mind." After completing the detection task for both passages, participants were asked whether they were able to do both letter searches in the manner instructed, and if not, what method they used instead.

\section{Results}

An ANOVA was conducted on the proportion of detection errors made out of the total possible. This analysis included the between-subjects factor of instruction type (auditory, visual) and the within-subject factors of passage (A, B) and word type (the, other words). Several other variables were included for control and counterbalancing purposes but were not included as factors in this or subsequent analyses. These variables were target order ( $t$ first, $h$ first), passage order (Passage A first, Passage $\mathrm{B}$ first), and target letter $(t, h)$. The ANOVA revealed no overall difference between Passage $\mathrm{A}(M=.07)$ and Passage $\mathrm{B}(M=.05),\left[F(1,22)=1.01, M S_{\mathrm{e}}=.0079\right.$, $p>.10]$, and no main effect of word type $[F(1,22)=2.85$, $\left.M S_{\mathrm{e}}=.0069, p>.10\right]$. However, importantly, there was a significant overall difference between the two types of instructions $\left[F(1,22)=4.63, M S_{\mathrm{e}}=.0256, p<.05\right]$, with the visual group making a greater proportion of errors (.09) than the auditory group (.02), and a marginally significant interaction of instruction group and word type $\left[F(1,22)=3.98, M S_{\mathrm{e}}=.0069, p<.06\right]$. This interaction is due to a greater proportion of detection errors on the common word the than on the other test words for the visual group, but a very small difference in the opposite direction, with a smaller proportion of errors on the than on the other test words for the auditory group (Table 1).

Separate planned ANOVAs of the two instruction groups were conducted. For the visual group the main effect of word type was significant $\left[F(1,11)=4.71, M S_{\mathrm{e}}=\right.$ $.0099, p=.05]$, whereas for the auditory group, it was not $[F(1,11)<1]$.

If participants are indeed following the auditory and visual memorization instructions, as suggested by the different pattern of results for the two instruction types on the detection task, they might also show a different pattern of responses on the preliminary memorization trials preceding detection. We conducted an exploratory analysis of the memorization trials from an earlier experiment (Schneider et al., 1989) and found that participants who reported seeing the words in the passage made fewer content-word or phrase substitutions than did participants who reported hearing the words in the passage. A possible explanation for this finding is that contentword or phrase substitutions result largely from a reconstruction process that occurs when participants visualize the scene described by the passage rather than the words in the passage. This reconstruction process would seem more likely to occur for participants hearing the words in the passage than for those seeing the words in the passage because it would be difficult to visualize the scene and the words at the same time. To determine whether the same pattern of results was found in the present experiment, in which participants were given explicit visual or auditory memorization instructions, we conducted an analysis of the content-word or phrase substitution errors in the five memorization trials of each passage preceding letter detection. An ANOVA was conducted on the 
number of content-word or phrase substitution errors, including the between-subjects factor of instruction type (auditory, visual) and the within-subject factor of passage (A, B). The ANOVA revealed a significant main effect of instruction type $\left[F(1,22)=7.65, M S_{\mathrm{e}}=2.4508\right.$, $p=.01]$, with auditory participants making more contentword or phrase substitutions $(M=1.7)$ than did visual participants $(M=0.5)$. The same pattern was found for both passages; neither the main effect of passage nor the interaction of instruction type and passage was significant $[F(1,22)<1$ in each case $]$.

\section{Discussion}

This experiment yielded strong evidence for flexibility in the type of memory representation used, with participants in the visual instruction group, but not those in the auditory instruction group, making a greater proportion of detection errors on the common word the than on other words containing a target. These findings suggest that participants generally have control over the memory representation they use to store text and that the chosen representation may profoundly affect what they can retrieve from memory. The memory instructions were repeated both before the initial presentation of the passage and before the detection task. The instruction type significantly affected the pattern of errors on the memorization trials preceding detection as well as the pattern of errors on the detection task. Participants in the auditory instruction group made more content-word or phrase substitutions during memorization than did participants in the visual instruction group. These results provide evidence that the instructions affected the memory representation used at the time of encoding as well as that used at the time of retrieval.

\section{GENERAL DISCUSSION}

Experiment 1 replicated the findings from Schneider et al. (1989) showing that participants differ in whether they report using a visual or an auditory memory representation for text. When searching for the target $h$, participants in the visual report group, but not those in the auditory report group, made significantly more detection errors on the very common word the than on the other, less common test words. Participants with high visual imagery were no more likely than those with low visual imagery to report a visual memory representation. Dividing participants by imagery ability did not yield any significant effects, also indicating that the memory representation chosen did not depend on imagery ability. In Experiment 2 the extent to which memory representation is flexible was explored. Participants were given instructions to use either a visual or an auditory memory representation, and participants in the visual instruction group, but not those in the auditory instruction group, made significantly more detection errors on the word the than on other test words. Choice of memory representation was, thus, shown to be generally flexible (although there were 5 additional individuals tested who reported using both methods of imaging for the detection task or reported using a method different from the one they had been instructed to use).

Because all participants we included in the analyses had met our memorization criterion, we ensured that all of these participants had al of the test words stored in memory at the time of the detection task. Nevertheless, there were large differences in retrieving the test words from memory as a function of the memory representation used by the participants. Thus, these experiments provide compelling evidence that the memory representation used to store text strongly influences what can be retrieved from memory about the text.

Finding that participants made more letter-detection errors on the word the than on other words with visual instructions not only supports the hypothesis that the participants used a visual memory representation in that case but also suggests that the representation they used contained units larger than a letter. Thus, the detection task used here can illuminate the units used to store text in memory, just as it has illuminated the units used in reading (see, e.g., Healy, 1976, 1994).
The memorization instructions affected the participants' pattern of errors on the memorization trials preceding letter detection as well as their pattern of errors on the detection task itself. We found that participants in the visual group made fewer content-word or phrase substitutions than did participants in the auditory group. Our explanation for this finding is that participants in the auditory group may reconstruct the words in the passage at least in part by visualizing the scene of the passage, whereas it would be difficult for participants in the visual group to use a similar reconstruction method because they are told to visualize the words

The text to be memorized by participants in this study was presented visually in all cases. In our earlier study (Schneider et al., 1989), we presented the text both visually and aurally, and found that the memory representation did not depend on the modality of the text ( $\mathrm{cf}$. Penney, 1989), but rather on some preference by the participants. In the present study, we found that the participants' preference for memory representation was not related to their imagery ability, but we do not know what factors did influence their preference.

The differences we found between auditory and visual memory representations support earlier findings of modality effects on long-term memory (see Penney, 1989, for a review). Unlike earlier studies, however, the differences we found cannot be attributed to stimulus input modality. As far as we know, our findings are unique in demonstrating a long-term modality effect achieved only through instructions concerning the modality of the memory representation. Nairne (1988) has proposed a model of memory that includes modality-dependent features that reflect perceptual aspects of stimuli, rather than sensory aspects, and that are a product of a language-analysis system. Nairne's model is thereby able to account for similar and interacting modality effects for auditory, lip-read, and mouthed stimuli. However, it is difficult to see how even this account could explain a modality effect achieved only through instructions, presumably because Nairne and Pusen (1984) were unable to obtain the standard modality effects in immediate serial recall by varying only imaging instructions.

Most important is our finding that the memory representation was flexible and influenced by experimenter instructions (i.e., it was under explicit control, contrary to recent arguments that strategy choice is largely implicit; see Reder \& Schunn, in press). The implication of this finding is that instructors may influence not only what is learned by students but also the type of memory representation used to store that information. Instructors should advise students to use the memory representation that would be most helpful given their particular subsequent memory needs. Because these needs are probably difficult to anticipate, it seems best, perhaps, to encourage students to adopt multiple forms of memory representation.

\section{REFERENCES}

Allen, P. A., Wallace, B., \& LoSchiavo, F. (1994). Influence of imaging ability on word transformation. Memory \& Cognition, 22. 565-574.

HEALY, A. F. (1976). Detection errors on the word the: Evidence for reading units larger than letters. Journal of Experimental Psychology: Human Perception \& Performance, 2, 235-242.

HEALY, A. F. (1994). Letter detection: A window to unitization and other cognitive processes. Psychonomic Bulletin \& Review, 1, 333-344.

MARKS, D. F. (1973). Visual imagery differences in the recall of pictures. British Journal of Psychology, 64, 17-24.

NAIRNE, J. S. (1988). A framework for interpreting recency effects in immediate serial recall. Memory \& Cognition, 16, 343-352.

NAIRNE, J. S., \& PUSEN, C. (1984). Serial recall of imagined voices. Journal of Verbal Learning \& Verbal Behavior, 23, 331-342.

PENNEY, C. G. (1989). Modality effects and the structure of short-term verbal memory. Memory \& Cognition, 17, 398-422.

Reder, L. M., \& SChUnN, C. D. (in press). Metacognition does not imply awareness: Strategy choice is governed by implicit learning and memory. In L. M. Reder (Ed.), Implicit memory and metacognition. Hillsdale, NJ: Erlbaum.

Schneider, V. I., Healy, A. F., Ericsson, K. A., \& Bourne, L. E., Jr. (1989). Letter detection errors in reading, auditory, and memory tasks. Journal of Memory \& Language, 28, 400-411. 


\section{NOTES}

1. An additional 10 individuals were tested, but their data were not analyzed because 2 of them were nonnative English speakers, 2 of them did not fully memorize the passage by the fifth repetition, 4 of them said they used both visual and auditory memory representations, 1 of them said she visualized the scene of the passage rather than the words in the passage, and 1 of them provided detection responses that were uninterpretable. The data from the final 3 individuals tested who reported using an auditory representation for the task were also not analyzed to equate the size of the groups.

2. An additional 16 individuals were tested, but their data were not analyzed because 4 of them were nonnative speakers of English, 5 of them reported using both methods of imaging for the detection task or reported using a method different from the one they had been in- structed to use (e.g., they said they used a visual method when instructed to use an auditory method), and 7 of them did not meet our memorization criterion for one of the passages. An additional 11 individuals were tested, but they did not provide data that could be analyzed because they failed to follow the detection-task instructions or because their detection responses were uninterpretable. Each of these additional individuals was replaced by another individual from the same condition and counterbalancing subcondition. The data from an additional 3 individuals tested (each of whom was the last one tested in a given counterbalancing subcondition) were also not analyzed to equate the size of the subconditions.

(Manuscript received April 5, 1995; revision accepted for publication February 1, 1996.) 